\title{
Study of variation of thermal diffusivity of advanced composite materials of E-glass fibre reinforced plastic (GFRP) in temperature range 5-300 K
}

\author{
KALOBARAN DAS, S M KAMARUZZAMAN, TAPAS RANJAN MIDDYA* and \\ SIDDHARTHA DATTA ${ }^{\dagger}$ \\ Department of Physics, ${ }^{\dagger}$ Department of Chemical Engineering, Jadavpur University, Kolkata 700 032, India
}

MS received 28 May 2008; revised 15 January 2009

\begin{abstract}
Modified Angstrom method is applied to study the variation of thermal diffusivity of plain woven fabric composite in closed cycle cryo-refrigerator (CCR) based set up in the temperature range 5-300 K. The set up used is plug in type and its small size offers portability. The set up works without use of any liquid cryogen. The temperature versus thermal diffusivity curve shows three distinct regions viz. 5-30 K, 30-120 K and $120-300 \mathrm{~K}$. In the first region thermal diffusivity varies exponentially and rapidly. In the second region thermal diffusivity changes exponentially but relatively slowly than that in the first region. In the last region the change in thermal diffusivity with temperature is exponential in nature but very slow.
\end{abstract}

Keywords. Thermal diffusivity; composite material; cryogenic temperature; phase difference; modified Angstrom method.

\section{Introduction}

With the advent of technology many newly emerging materials such as fibre reinforced plastic (FRP) are coming up with very attractive mechanical properties like low weight and high tensile strength non-corrosive character (Mallick 1997), but the thermal application of these materials are being restricted due to lack of reliable thermo physical data. The major reasons of non-availability of these kind of data is lack of proficient set up that can measure the thermo physical properties of said materials. The successful design in the low temperature system demands that the cool down process should be fast and smooth. Thus the experimental data of thermal diffusivity of composite materials is of considerable support to optimize the cryo system performance, heat transfer and cool down process analysis. Keeping above facts in mind a CCR based setup has been developed. In most of the studies liquid cryostat has been used for production of low temperature. The major problem with this kind of setup is the difficulty in smooth transition from one set point to other set point temperature. The theoretical basis of this setup is modified Angstrom method. The literature review shows that the study of variation of thermal diffusivity with temperature in the range $5-300 \mathrm{~K}$ has been done by only a few workers (Zavaritsky 1959; Tomokiyo and Okada

*Author for correspondence (middya@phys.jdvu.ac.in)
1968; Vandersande and Phol 1980; Evseeva and Tanaeva 1995, 1998; Kalaprasad et al 2000). The setup developed (figure 1) has been designed, fabricated, assembled and tested for calibration to evaluate the thermal performance of advanced composite materials down to $5 \mathrm{~K}$ without the use of any cryogen.

\section{Sample used and its preparation}

E-glass fibre reinforced epoxy resin composite sample has been prepared for the purpose of present study. The constituents of the E-glass fibre used has been given in table 1. The epoxy resin (LY-556) along with hardener (HY951) used here worked as matrix and E-glass fibre as reinforcement. Besides few limitations like high cost, the advantages of using epoxy as matrix includes (i) it offers

Table 1. Composition of E-glass fibre

\begin{tabular}{lc}
\hline Constituent & Quantity $(\%)$ \\
\hline $\mathrm{SiO}_{2}$ & $55 \cdot 2$ \\
$\mathrm{Al}_{2} \mathrm{O}_{3}$ & $14 \cdot 8$ \\
$\mathrm{~B}_{2} \mathrm{O}_{3}$ & $7 \cdot 3$ \\
$\mathrm{MgO}$ & $3 \cdot 3$ \\
$\mathrm{CaO}$ & $18 \cdot 7$ \\
$\mathrm{~K}_{2} \mathrm{O}$ & $0 \cdot 2$ \\
$\mathrm{Na}_{2} \mathrm{O}$ & $0 \cdot 2$ \\
$\mathrm{Fe}_{2} \mathrm{O}_{3}$ & $0 \cdot 2$ \\
$F_{2}$ & $0 \cdot 1$ \\
\hline
\end{tabular}


greater control over cross linking process which helps in increasing the strength and ductility of the material and (ii) it is water and temperature resistant and suffers negligible shrinkage during curing.

The diameter of the samples is of $18.0 \mathrm{~mm}$ and their thickness varies from $1.0 \mathrm{~mm}$ to $1.25 \mathrm{~mm}$ with different volume fractions.

About 10 bar pressure was applied for the preparation of void-less specimen. The pressure is applied on the dye during curing which helps the fabric to be in proper position along with the component during the transition from liquid to solid. The direction of spread of fibre is in parallel with the diameter.

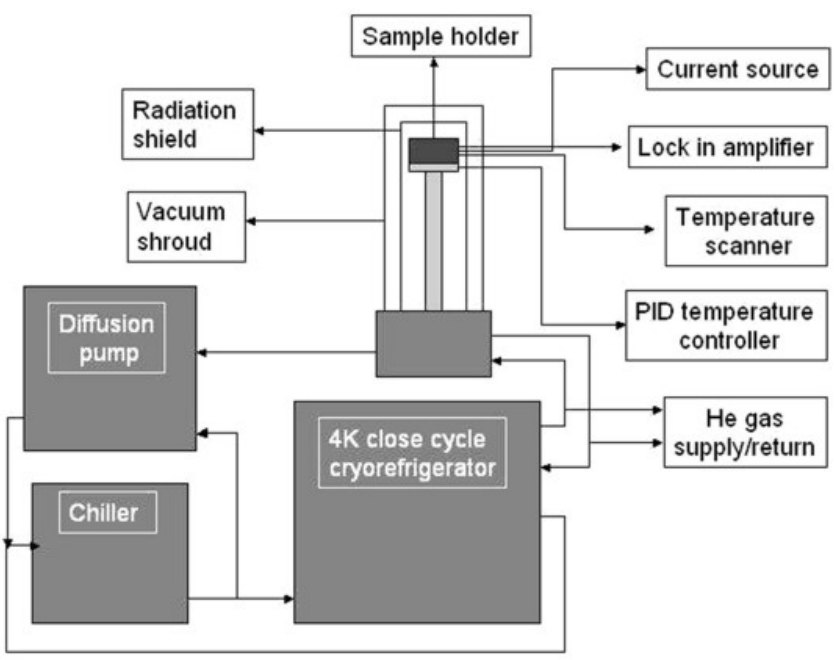

Figure 1. Schematic diagram of experimental set up for measuring thermal diffusivity.

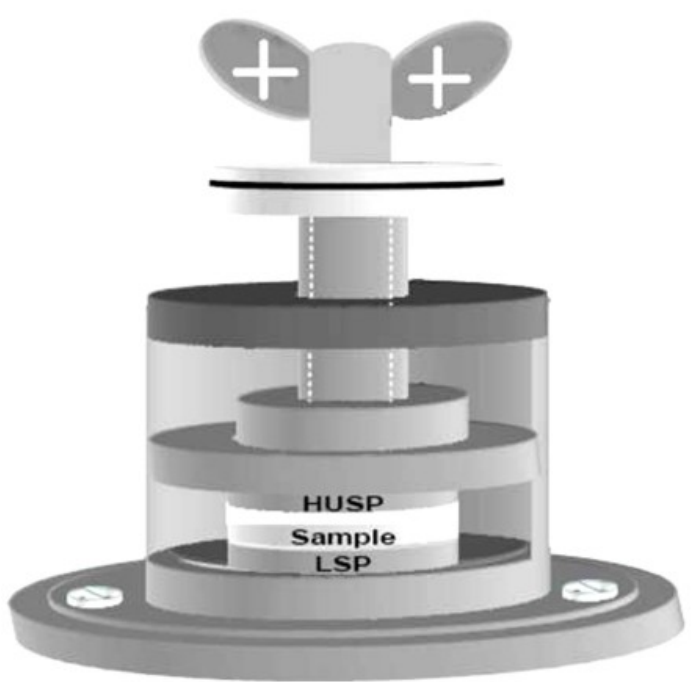

Figure 2. Diagram of sample holder for measuring thermal diffusivity (HUSP: heater cum upper sensor plate and LSP: lower sensor plate).

\section{Experimental}

\subsection{Theory}

In modified Angstrom method sample is heated periodically at one end, due to which a temperature wave is propagated along the length of the sample with the same period but with diminishing amplitude. During the pas-

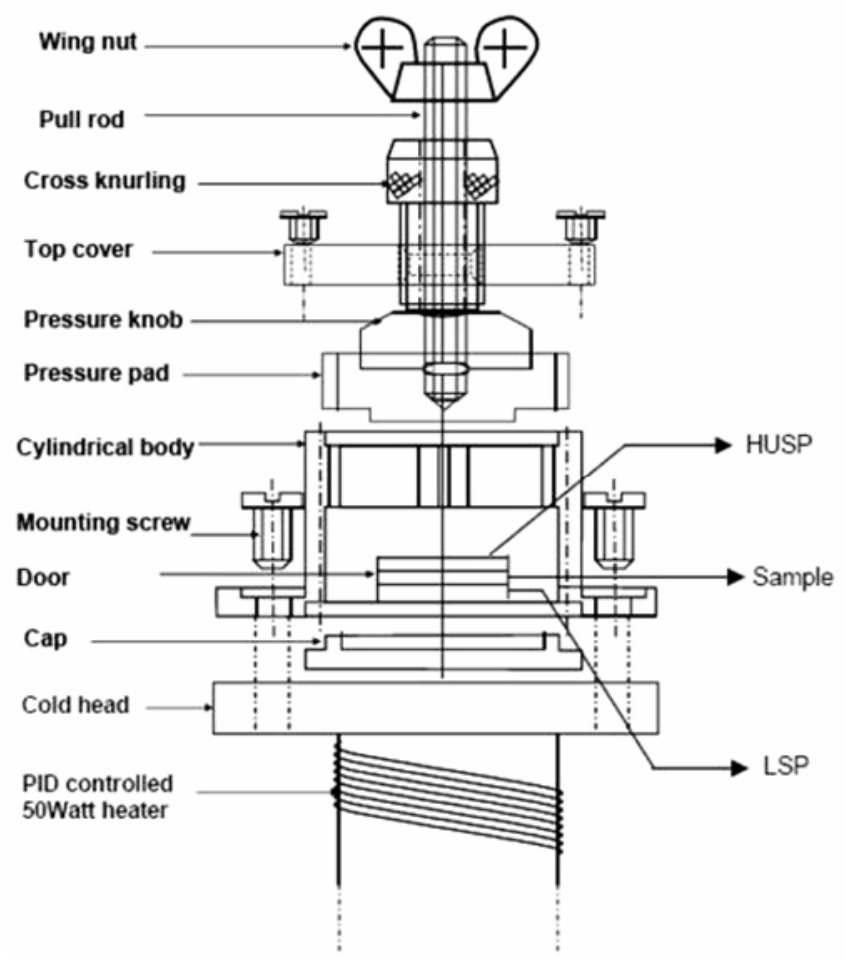

Figure 3. Diagram of sample holder fixed on the cold head.
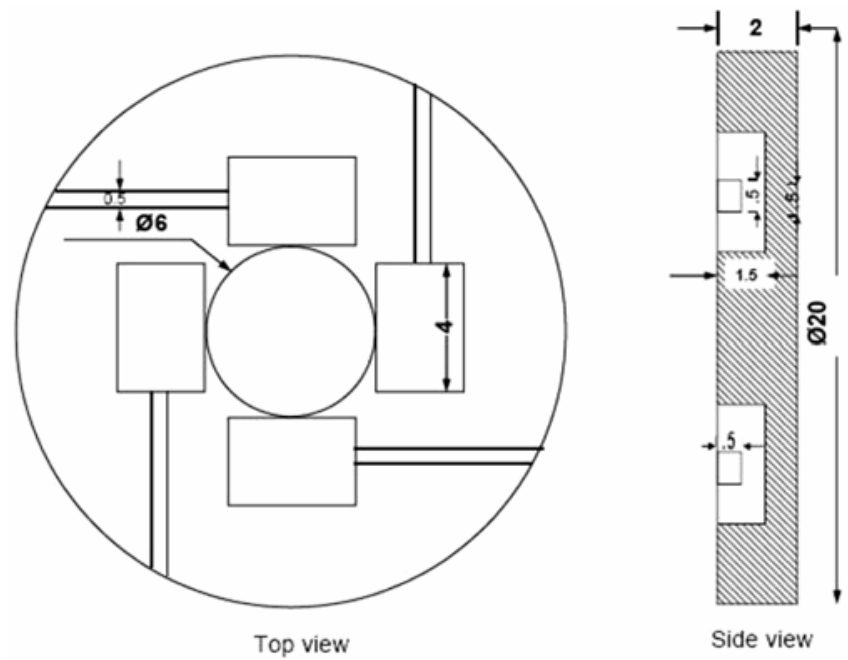

Figure 4. Diagram of the heater cum sensor plate. Face shown in the figure will be in contact with sample. Heater wire is wound around this sensor plate and hence forming heater cum sensor plate (all the dimensions given in $\mathrm{mm}$ ). 
sage through the sample the heat wave suffers a change in phase. So there exists a phase relationship between any two points across the length of the sample. From the phase relationship the phase difference between two points can be determined. Considering appropriate boundary condition in solving the Fourier heat transfer equation for heat wave we can reach the expression for thermal diffusivity $(D)$ of the kind stated below (Kogure and Hiki 1973).

$$
D=\frac{\pi \ell^{2}}{T(\Delta \phi)^{2}},
$$

where $\ell$, the length of the sample and $T=2 \pi / \omega$, the time period of the temperature wave. $\omega$ is the frequency of the

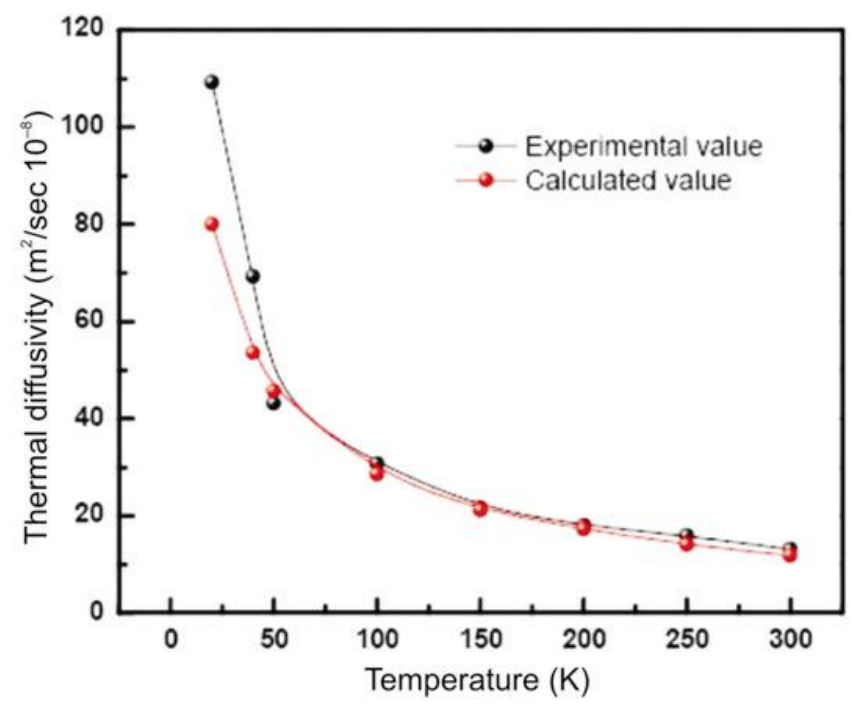

Figure 5. Variation of thermal diffusivity with temperature of Teflon.

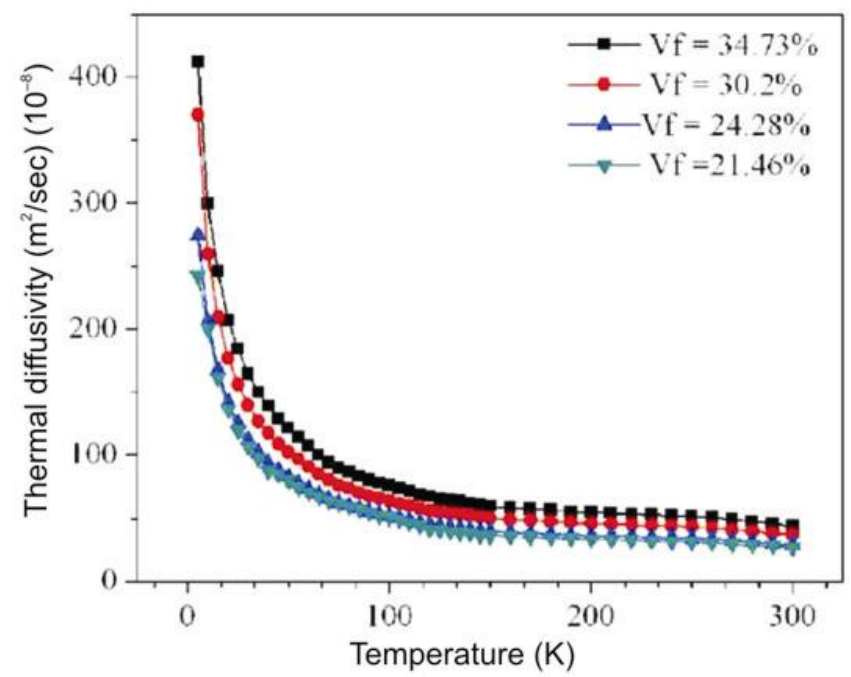

Figure 6. Variation of thermal diffusivity with temperature of the E-glass/epoxy woven fabric composites having different volume fractions $\left(V_{\mathrm{f}}\right)$ of fibre. The solid lines are best fitting with third order exponential decay equation. propagating wave, $\Delta \phi$ the phase lag suffered by the propagating heat wave in passing two points, $\ell$, distance apart.

\subsection{The set up}

The major components of the set up used are a closed cycle cryo-refrigerator, a sample holder, a temperature monitor, PID temperature controller, sensor and a lock-in amplifier. The set up developed, can measure thermal diffusivity in the temperature range $5-300 \mathrm{~K}$, is calibrated by the standard result of Teflon (figure 1). To restrict different modes of heat in leak, sample holder is insulated by vacuum at the order of $10^{-6}$ mbar created by rotary and diffusion pump assembly and covered with stainless steel radiation shield. The cold head temperature is controlled by a lakeshore make model 332 PID temperature controller via a calibrated silicon diode sensor with a sensitivity of $0.01 \mathrm{~K}$ up to $40 \mathrm{~K}$. Below $40 \mathrm{~K}$ the fluctuation in cold head temperature is $0 \cdot 2-0 \cdot 3 \mathrm{~K}$. This set up takes $40-$ $50 \mathrm{~min}$ to reach a new set of temperature at loaded condition. For the study of disc shape sample a special type of sample holder has been developed (figures 2, 3). The sample within the sample holder is sandwiched between the sensor plates (figure 4).

For measuring the phase difference two sets of sensor developed (Kamaruzzaman et al 2008) are impregnated in two sensor plates placed on the two sides of the sample, one of the sensor plates being used as core of the heater. The heater is made up of the insulated copper wire coil whose resistance at room temperature is $\sim 15 \mathrm{ohm}$. To keep the sample heater sensor plate in proper position, sample-heater-sensor assembly is pressed to the base of the sample holder by the pressure putting screw. The heater is fed by a constant a.c. current source. The supply

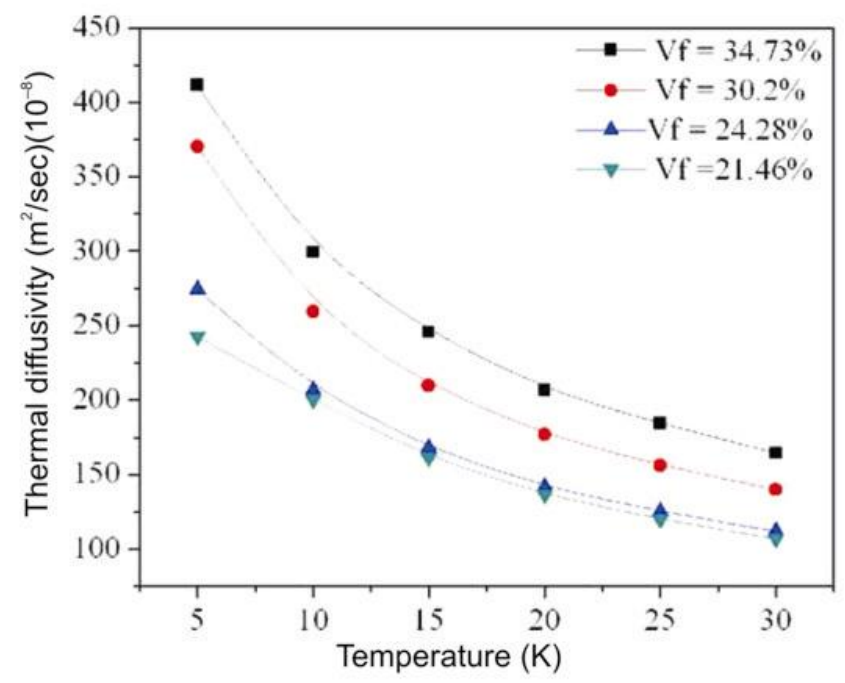

Figure 7. Variation of thermal diffusivity with temperature range $5-30 \mathrm{~K}$ of different volume fractions. 


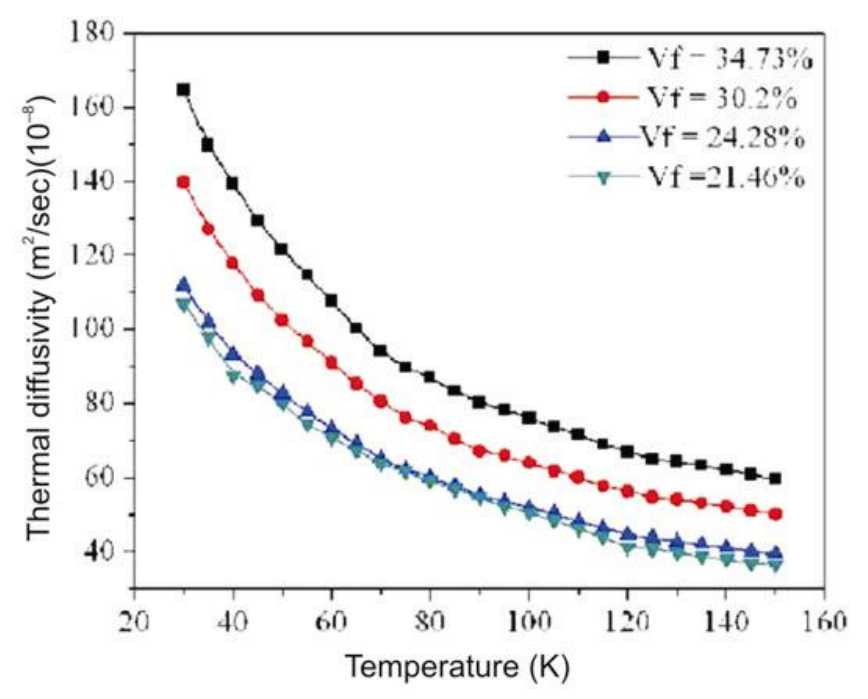

Figure 8. Variation of thermal diffusivity with temperature range $20-160 \mathrm{~K}$ of different volume fractions.

current has rms value of around $50 \mathrm{~mA}$ in $300 \mathrm{~K}$ and around $90 \mathrm{~mA}$ in $5 \mathrm{~K}$. Due to this a.c. current heater generates a heat wave of frequency double than that of supplied current. SRS make model 830 dual phase lock in amplifier has been used to measure the difference in phase across the sample.

\section{Results and discussion}

Teflon has been used as standard sample for the calibration (figure 5) of the sample. The thermal diffusivity of the Teflon has been calculated from available thermal conductivity and heat thermal capacity data using the equation

$$
D=\frac{k}{\rho c},
$$

where $D$ is the thermal diffusivity, $k$ the heat conductivity and $\rho$ the thermal capacity per unit volume. The departure of the experimental results from the theoretical value (Timmerhaus and Flynn 1989) is around 15\%. The reproducibility of the set up has been confirmed within a variation of $15 \%$.

The temperature dependence of thermal diffusivity for different volume fractions has been shown in figures 6-8. It shows three distinct regions: within the temperature range $5-30 \mathrm{~K}$, thermal diffusivity changes exponentially with high slope with temperature, in the intermediate range of $30-120 \mathrm{~K}$ thermal diffusivity changes exponentially with moderate slope and in the high temperature range of $120-300 \mathrm{~K}$ again the thermal diffusivity varies slowly in exponential manner. This is why total experimental temperature zone i.e. $5 \mathrm{~K}-300 \mathrm{~K}$, has been divided in three zones viz. $5-30 \mathrm{~K}, 30-120 \mathrm{~K}$ and $120-300 \mathrm{~K}$. The probable reason behind the change in rate of thermal diffusivity with respect to temperature may be the resonating change in phonon dispersion (Evseeva and Tanaeva 1998).

\section{Conclusions}

The observed reduction in thermal diffusivity with increase in temperature may be the effect of shortening of the mean free path of the phonons with the increase in temperature. In other words, it may be said that as the temperature increases the mean free path of the phonon in the solid decreases and in turn the thermal conductivity decreases and specific heat increases. As the thermal diffusivity is directly proportional to the thermal conductivity and inversely proportional to the specific heat so the increase in temperature results in decrease in thermal diffusivity. The increase in thermal diffusivity with increasing fibre concentration may be due to short-circuiting within the woven fabrics and enhancement in fibre surface.

\section{Acknowledgement}

The authors are grateful to Prof. A Bhowal and Mr Ashim Kumar Chatterjee, Jadavpur University, Kolkata, for their valuable suggestions and help.

\section{References}

Evseeva L E and Tanaeva S A 1995 Cryogenics 35277

Evseeva L E and Tanaeva S A 1998 Cryogenics 38253

Kalaprasad G, Pradeep P, Mathew G and Thomas S 2000 Compos. Sci. \& Technol. 602967

Kamaruzzaman S M, Das K, Middya T R and Datta S 2008 Indian J. Cryogenics (communicated)

Kogure Y and Hiki Y 1973 Jap. J. Appl. Phys. 12814

Mallick P K 1997 Composites engineering handbook (New York: Macel Dekker Inc)

Timmerhaus D and Flynn T M 1989 Cryogenic process engineering (New York: Plenum Press)

Tomokiyo A and Okada T 1968 Jap. J. Appl. Phys. 7128

Vandersande J W and Phol R O 1980 J. Rev. Sci. Instrum. 52 1694

Zavaritsky N V 1959 Progress in cryogenics (eds) K Mendelssohn (London: Heywood \& Company Ltd.). 1 p. 209 